\title{
DIESEL GAS DUAL ENGINE WITH LIQUID LPG INJECTION INTO INTAKE MANIFOLD
}

\author{
Ales Dittrich, Stanislav Beroun, Tomas Zvolsky \\ Technical University at Liberec, Czech Republic \\ ales.dittrich@tul.cz, stanislav.beroun@tul.cz,tomas.zvolsky@tul.cz
}

\begin{abstract}
This paper presents an experimental research on the fuel control system which is installed on the diesel engine Cummins to assess the technical characteristics and emissions, when the engine is using dual fuel liquid LPG-Diesel with different ratios of LPG. The experimental results show that the engine running dual fuel LPG - Diesel reached $96 \%$ maximum moment. Average torque and average power were reduced, but not significantly compared to the case of the engine using $100 \%$ diesel. The percentage of the alternative LPG fuel could be reached by $60 \%$. For engine using dual fuel, the concentration of $\mathrm{CO}_{2}$ and particle concentration are decreased.
\end{abstract}

Keywords: dual engine, diesel, LPG, exhaust emission.

\section{Introduction}

The dual compression ignition (CI) engine (LPG gas and diesel) is operated simultaneously on diesel and LPG. This engine has some specific features against the diesel engine, e.g., the engine can only be started on diesel and can only be converted to dual operation after short-term engine warm-up. In dual operation, the dual engine control system must regulate the gaseous fuel dose and the injected diesel dose, so that the combustion course of the homogeneous LPG-air mixture has a sufficient reserve against detonation burning (so-called "knocking"). The risk of "knocking" increases with the increasing richness of the LPG and air mixture and decreases, when the temperature of the intake mixture to the engine is reduced. Injection of liquid LPG into the pipeline behind the charge air cooler (coupled with cooling the mixture) allows for increased LPG share in dual engine operation. With a greater reduction in the fuel injected, an additional risk of dual engines is overheating of the injection nozzles: the dual-engine control system must therefore ensure reliability of operation also from this point of view.

\section{Materials and Methods}

The dual engine is installed at a test facility at the Department of Vehicles and Engines at the Technical University of Liberec. The test station is equipped with a Schenck D700 hydraulic dynamometer. The dynamometer is controlled by the Schenck system with the ControlWeb control system under the Windows operating system, which allows the engine to operate according to a programmed test mode with electronic data collection of all standard engine operating variables. Special measuring equipment: MGC Hottinger (for thermocouple fast data collecting), AVL Indimeter 619 (indicating the pressure inside the combustion chamber), Horiba Mexa-One (for gaseous exhaust emissions), and Engine Exhaust Particle Sizer (PM) EEPS TSI 3090 were also used for the measurement.

Table 1

Engine parameters specified for laboratory measurements [1]

\begin{tabular}{|c|c|}
\hline Type & $\begin{array}{c}\text { CI, 4 cylinder inline, } \\
\text { turbocharged and } \\
\text { aftercooled }\end{array}$ \\
\hline Bore X Stroke & $107.61 \mathrm{X} 123.7 \mathrm{~mm}$ \\
\hline $\begin{array}{c}\text { Swept volume of } \\
\text { engine }\end{array}$ & $4.497 \mathrm{~cm}^{3}$ \\
\hline Maximum torque & $760 \mathrm{Nm}$ \\
\hline Compression ratio & $17.3: 1$ \\
\hline Cooling system & water \\
\hline
\end{tabular}

The LPG fuel system is made from the components of VIALLE (pressure fuel pump with a pump, fuel distribution with a pressure regulator and injection solenoid valves). The LPG injector end parts are self-constructed (with electric outlet nozzle heating and minimal flow rate reduction in the engine 
intake manifold), the production of injector end parts is also proprietary (TUL) [1-4]. The assembly of the injection solenoid valves and the end part of the injector with the heating of the LPG outlet nozzle is shown in Fig. 1, the installation of the LPG injectors into the pipeline is shown in Fig. 2.
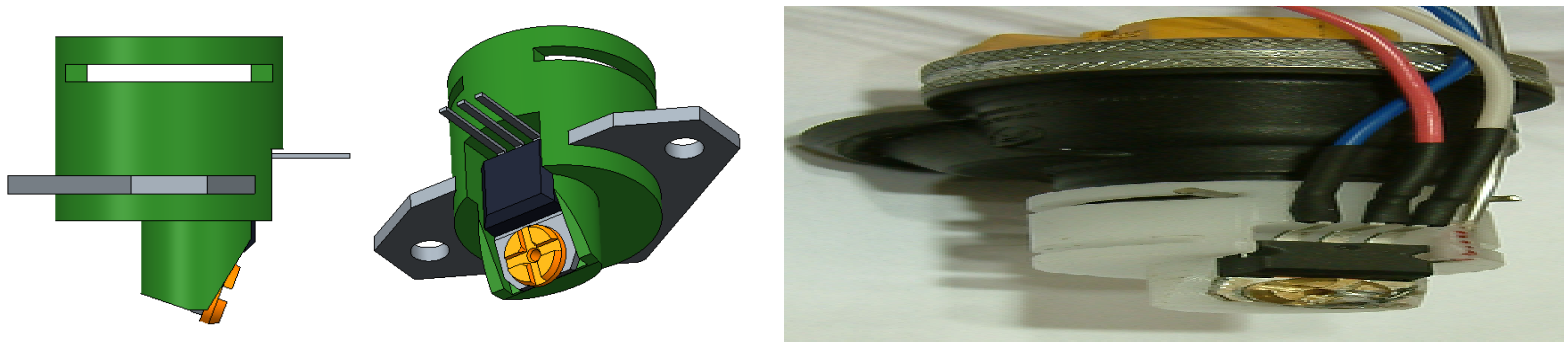

Fig. 1. Assembly of injection solenoid valves and end part of injector with heating of LPG outlet nozzle

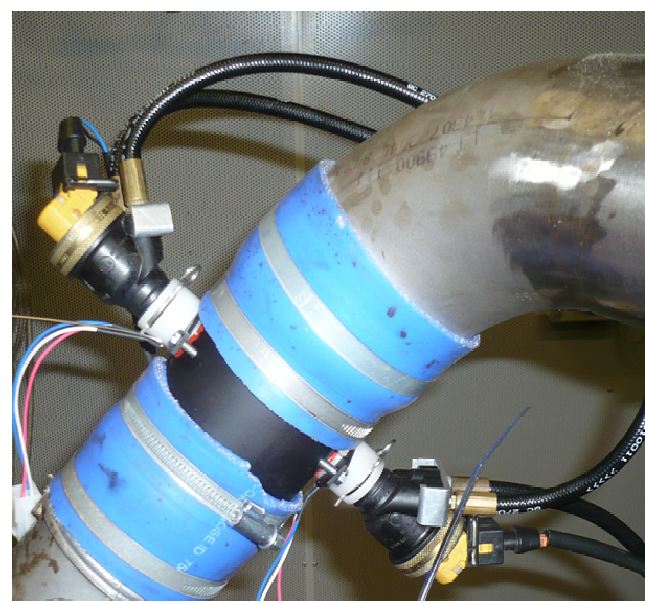

Fig. 2. Installation of LPG injectors into pipeline

LPG injectors located in the pipeline behind the charge air cooler before the engine filling line ("brown" injection valves with the flow rate number 25) have been used for dual operation. Liquid LPG injection is controlled, depending on the operating mode of the engine, by the control SW of the SOLARIS Diesel + LPG control unit, connecting diesel fuel systems (diesel) and auxiliary fuel gas (LPG). The control software of the SOLARIS electronic control unit (ECU) reduces the proportion of diesel and replaces it with LPG in the engine for the entire engine operating range (the graph on the monitor above shows the LPG share). After verifying the dual-engine properties of the vehicle in realtime operation, the data are stored in the ECU control program. Due to the experimental research on a functional sample of the CUMMINS gas dual engine, the data for dual engine operation were not stored in SOLARIS ECU memory.

The CUMMINS gas dual-engine is equipped with a set of sensors that provide important information for a comprehensive evaluation of the engine power, energy, thermodynamics and emission parameters, when comparing the engine measurements on both diesel and dual diesel + LPG. Figure 3 shows the exhaust side of the engine, with thermocouples for exhaust gas temperature measurement at the output from each engine cylinder (significant in terms of quality control of the mixture in individual cylinders) and in the front of the turbine.

The proportion of LPG was determined according to the measured total fuel consumption during diesel operation. The calculation determined the thermal input of the engine in the fuel and with insignificant simplification it can be assumed that in dual operation the engine will have the same overall efficiency and hence the same thermal energy input in the fuel as in diesel only. Engine operating states in dual mode are the result of power consumption in diesel and LPG power consumption. The measurement was performed by setting the torque value in diesel mode only (for diesel consumption [5], the heat input was determined according to consumption) and then the torque increased by the LPG input. The increase in torque in dual operation is then due to an increase in the 
thermal input in the fuel to the engine, and this thermal input for engine operation at given speeds and torque is known (with the above assumption) for engine-only diesel measurements. The LPG shares then represent the proportion of LPG in the total heat input in the fuel to the engine.

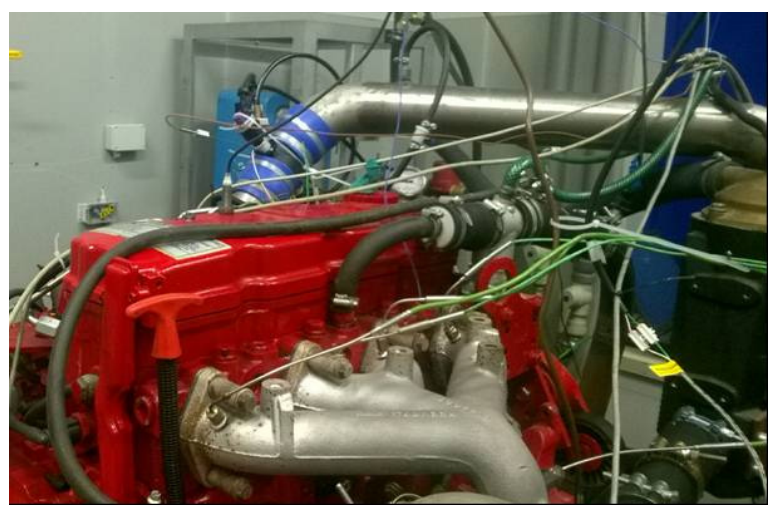

Fig. 3. View of engine at test site

\section{Results and discussion}

Figure 4 shows the engine operating conditions in diesel + LPG dual mode in low, medium and high revolution load modes. Depending on the overall fuel consumption during diesel operation the thermal input of the engine in the fuel (black line) is determined by conversion. With insignificant simplifications, it can be assumed that in dual operation the engine will have the same overall efficiency and hence the same thermal energy input in the fuel as when running on diesel. Dual engine operating states (red arrows) result from power consumption in diesel (red horizontal lines) and power consumption in LPG (red vertical lines). The allowable increase in LPG power (i.e. the increase in the engine torque in dual operation above the torque setpoint when operating on diesel) was determined by checking the combustion pressure of the cylinder (only very weak detonation combustion knocking allowed).
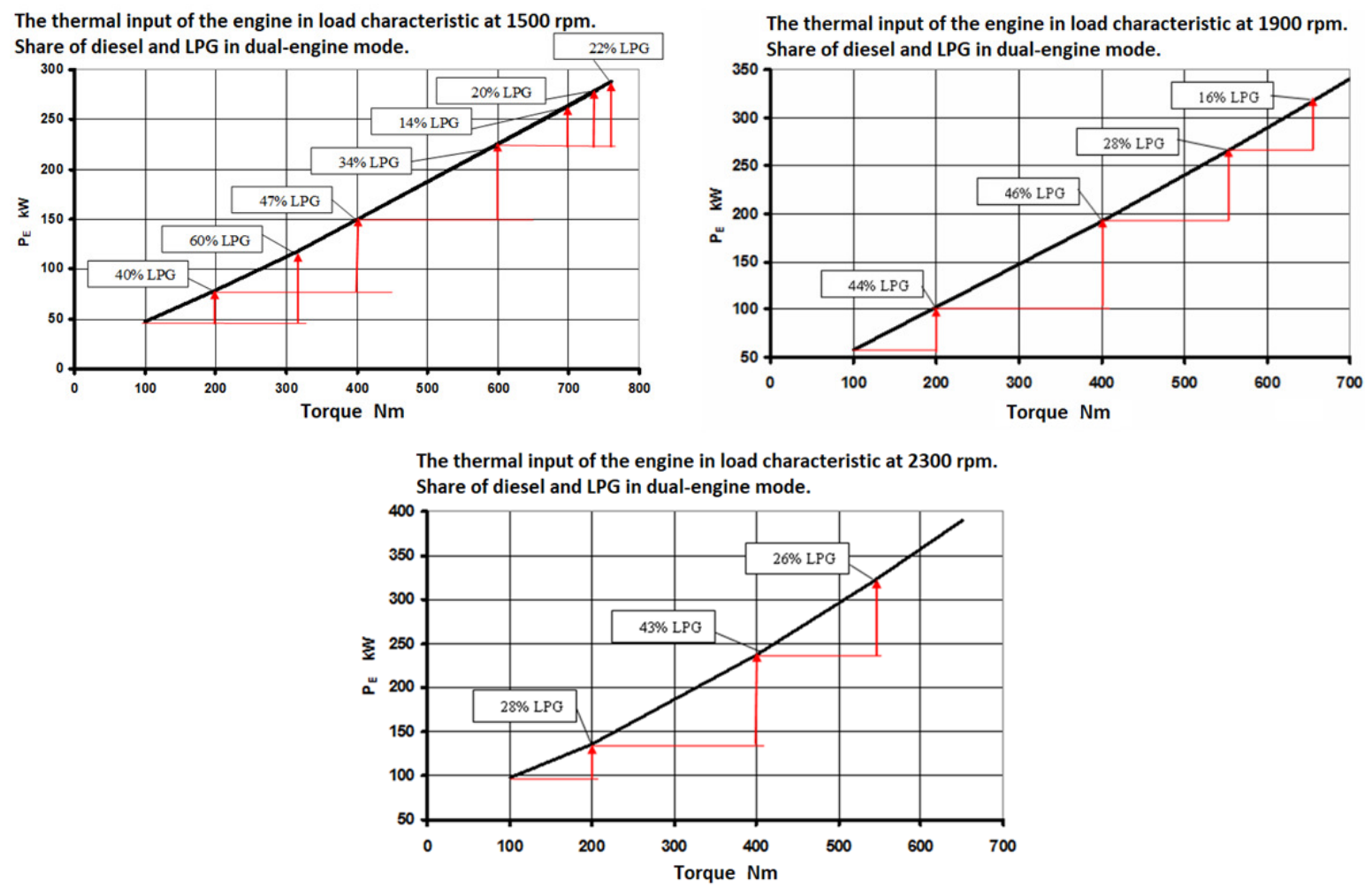

Fig. 4. Thermal input of engine in load characteristics 
Figure 5 shows the exhaust temperature in load characteristic modes for diesel and dual - engine operation. Lower exhaust gas temperatures in dual operation indicate a higher efficiency of the thermal energy conversion of the cylinder load in the mechanical work in the engine (a more favorable pressure pattern in the engine cylinder, when combusting a flammable LPG mixture), but also the differences in the fabric properties of the flue gases from the diesel and the mixture of LPG and diesel.

Exhaust temperature - load characteristic at $1500 \mathrm{rpm}$.

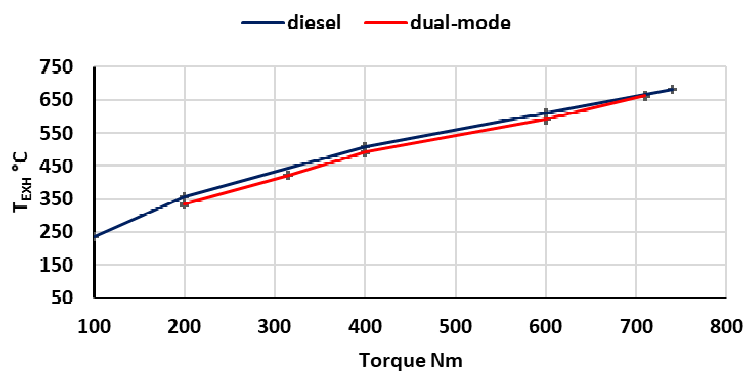

Exhaust temperature - load characteristic at $1900 \mathrm{rpm}$.

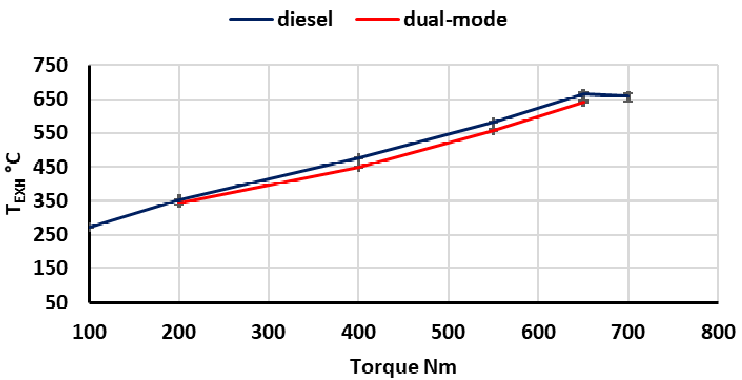

Exhaust temperature - load characteristic at $2300 \mathrm{rpm}$.

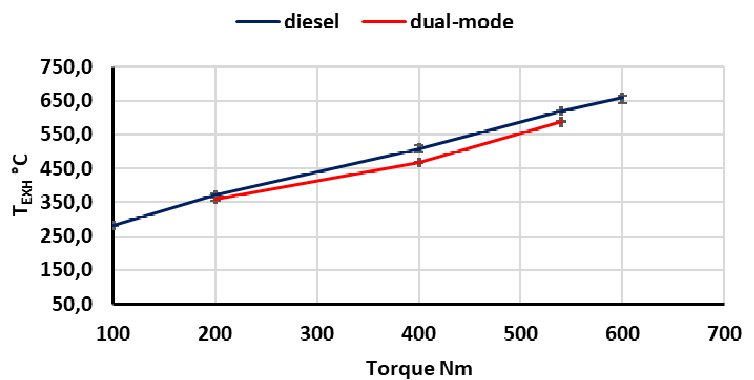

Fig. 5. Exhaust temperature in load characteristics

Figure 6 shows combustion pressures in load characteristic modes for diesel and dual - engine operation, as determined by high pressure indication (mean values - arithmetic maximum cylinder pressures in 100 consecutive engine operating cycles).

Combustion pressure - load characteristic at $1500 \mathrm{rpm}$.

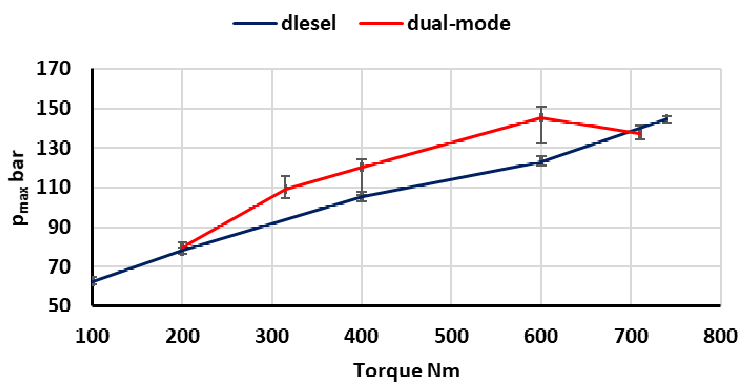

Combustion pressure - load characteristic at $1900 \mathrm{rpm}$.

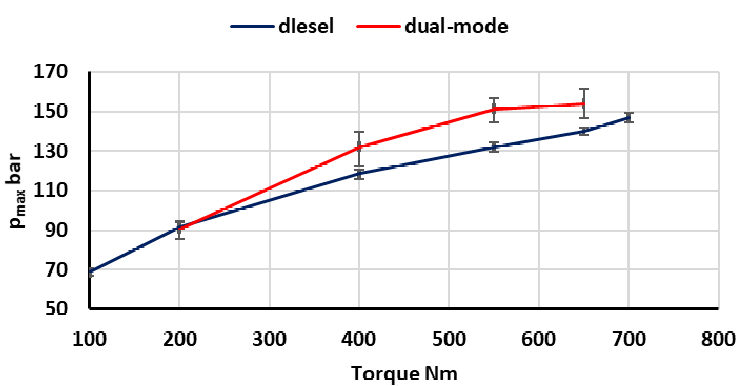

Combustion pressure - load characteristic at $2300 \mathrm{rpm}$.

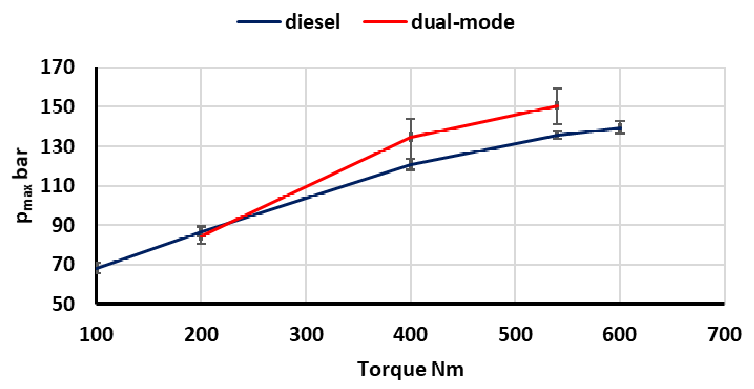

Fig. 6. Combustion pressure in load characteristics 
Higher combustion pressures in dual engine operation are the result of sparking a mixture of about $10-14^{\circ}$ before the TDC after ignition of a very small pre-fuel injection rate and a rapid burning of the LPG mixture. Increased combustion pressures in dual engine operation against combustion pressures during diesel engine operation are not a risk to the robust diesel engine design - shown in Fig. 7.

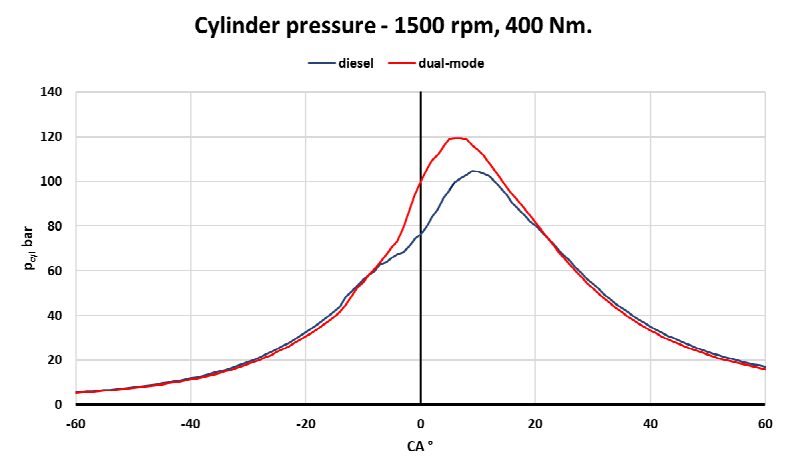

Fig. 7. Cylinder pressure 1500 rpm, 400 Nm (47 \% LPG)

In dual engine operation modes, the $\mathrm{CO}_{2}$ concentration in the exhaust gas is lower than that of the diesel engine only. This is the result of the lower carbon content in $1 \mathrm{~kg}$ of LPG (the carbon content in $1 \mathrm{~kg}$ of diesel) and the higher efficiency of heat energy conversion to mechanical work in the engine due to the kinetic combustion of homogeneous gaseous fuel mixture with air (against diffuse combustion heterogeneous diesel injected) - shown in Fig. 8.

$\mathrm{CO}_{2}$ concentration - load characteristic at $1500 \mathrm{rpm}$.

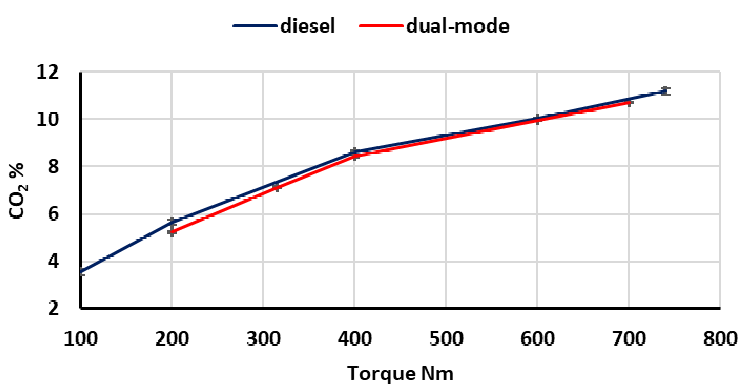

$\mathrm{CO}_{2}$ concentration - load characteristic at $1900 \mathrm{rpm}$.

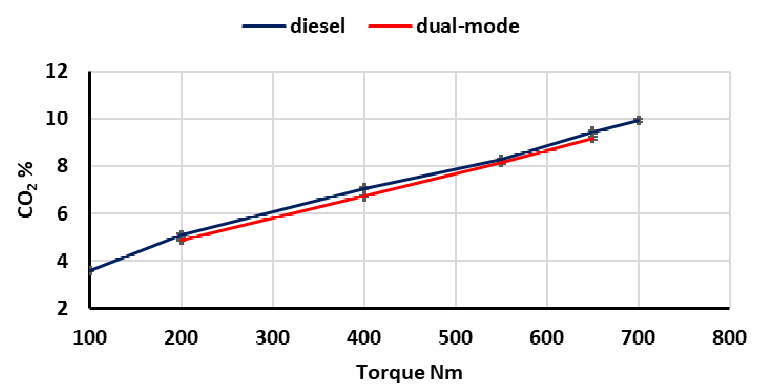

$\mathrm{CO}_{2}$ concentration - load characteristic at $2300 \mathrm{rpm}$.

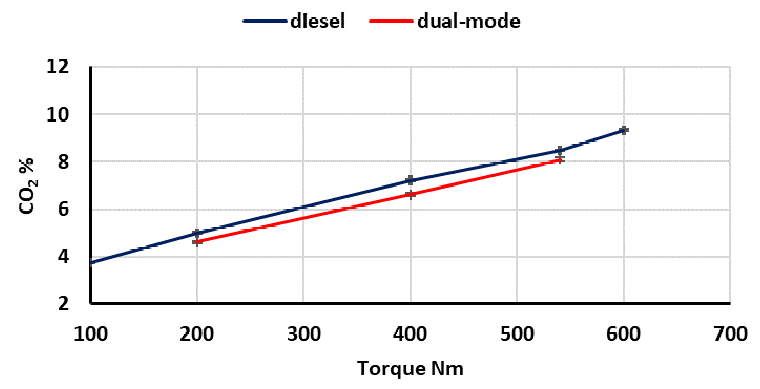

Fig. 8. $\mathrm{CO}_{2}$ concentration in exhaust gas

Figure 9 shows total sum of particle emission in the observed particle size spectrum. It shows lower total concentration, when operating in dual - mode compared to diesel engine operation. 

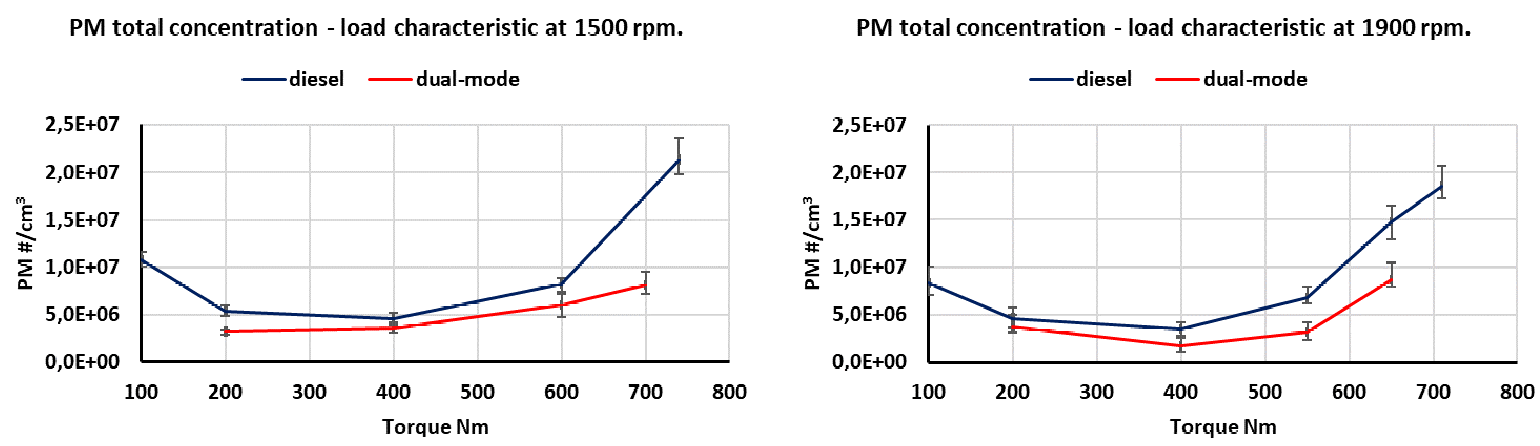

PM total concentration - load characteristic at $2300 \mathrm{rpm}$.

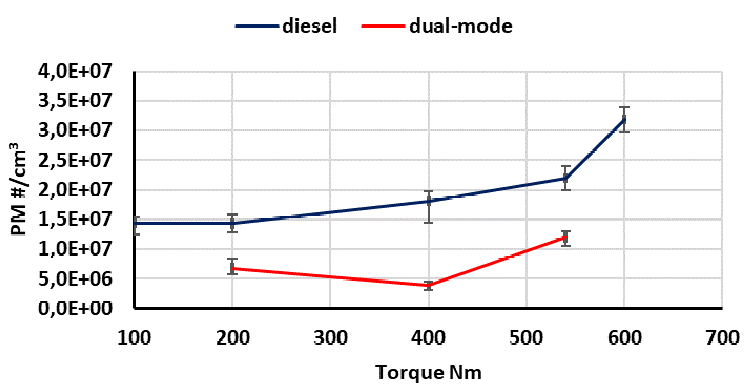

Fig. 9. PM total concentration - load characteristics

\section{Conclusions}

The paper shows experimental research and measurements on a modified diesel engine for dual mode operation (diesel + LPG). The results also show that, in the medium loads in each single load characteristic, almost half of the injected fuel can be replaced by LPG, which, despite the increase in the maximum pressures in the combustion chamber (but with the same performance parameters as for diesel only), has a positive effect on the concentration of $\mathrm{CO}_{2}$ and particle concentration in the exhaust gases. The results in the graphs in Fig. 4 to 9 show, while maintaining the same performance parameters as for diesel only, a reduction in $\mathrm{CO}_{2}$ emissions and a reduction in the total particle concentration of the exhaust gas.

\section{Acknowledgements}

The results of this project LO1201 were obtained through the financial support of the Ministry of Education, Youth and Sports in the framework of the targeted support of the "National Programme for Sustainability I" and the OPR\&DI project Centre for Nanomaterials, Advanced Technologies and Innovation CZ.1.05/2.1.00/01.0005.

\section{References}

[1] Beroun S., Brabec P., Dittrich A., Dráb O., Nguyen Thanh T. Computational Modeling of the liquid LPG Injection into the suction Manifold of an SI Vehicle Engine, in: Proc. 4th International Conference on Mechanical and Aerospace Engineering, 2013 Moscow, ISSN 1660-9336, pp. 355-359. Journal of Applied Mechanics and Materials, 390, pp. 355-359. ISSN 1660-9336 (http://www.scientific.net/AMM.390.355).

[2] Nguyen Thanh T. Injection of liquid LPG into the intake manifold of the engine. Doctoral Thesis, 2013, Technical University of Liberec.

[3] Cengel Y. A., Boles M. A. Thermodynamics, 2008, in: Proc. 6th edition. McGrow - Hill Comp., New York, ISBN 978-0-07-352921-9.

[4] Kim H., Im J. K., Lee J. H., Park J. N. An Apparatus for Preventing Icing of Injector for LPG Vehicle [patent]. 2007, KR 100757123. 10.9.2007.

[5] Scholz C. etc. Dynamická měření systému Common Rail na motoru CUMMINS ISBe4. Technická zpráva KVM TU v Liberci, SM 622/2010. 